\title{
Photonic band structure of Sierpinski waveguide networks
}

\author{
Maohua Li \\ Department of Physics, South China University of Technology, Guangzhou 510640, China \\ Youyan Liu* \\ Chinese Centre of Advanced Science and Technology (World Laboratory), P.O. Box 8730, Beijing 100080, China \\ and Department of Physics, South China University of Technology, Guangzhou 510640, China \\ Zhao-Qing Zhang \\ Department of Physics, Hong Kong University of Science and Technology, Clear Water Bay, Kowloon, Hong Kong, China
}

(Received 24 September 1999)

\begin{abstract}
The photonic band structure and transmission properties of Sierpinski fractal networks (SNs) made of one-dimensional waveguides are studied with the generalized eigenfunction method. In the absence or presence of dissipation and in different exit situations, we have numerically calculated the transmission coefficient as a function of frequency in the range $0-500 \mathrm{MHz}$ for the first four generations of SNs. As the number of generations is increased, the structures in the transmission spectra show explicitly the evolution of discrete eigenmodes and the corresponding photonic band gap structures in such fractal networks. The gap structures are not altered by the presence of dissipation and are independent of the exit situation. An interesting anomaly due to dissipation is found at certain frequencies where a resonant peak is split into two peaks with zero transmission in the valley.
\end{abstract}

\section{INTRODUCTION}

In the last decade, the localization of classical waves in random media has been under intensive study. ${ }^{1}$ Unlike electron localization, the localization of classical waves is purely a result of multiple scattering in a random environment and free from the complications arising from interaction effects. However, due to Rayleigh scattering at low frequencies, it is more difficult to localize classical waves than to localize electrons. Photonic band gap (PBG) materials have also been greatly studied during the past decade. ${ }^{2-4}$ The existence of gaps in photonic crystals where electromagnetic waves are forbidden has important implications in both fundamental science and technological applications. It has been pointed out that it is easier to observe localized states inside the band gap of a disordered PBG system. ${ }^{3}$ The localization of electromagnetic waves has been observed in one-dimensional (1D) and 2D PBG materials. ${ }^{5,6}$ For 3D systems, efforts have been focused only on wave localization in random media. The effects arising from wave localization have been reported in microwave experiments, ${ }^{7}$ although direct interpretation of localization was complicated by the presence of large absorption. Direct evidence of light localization has also been reported in strongly scattering media of semiconductor powders based on the size dependence of the transmission coefficient. ${ }^{8}$ Very recently Zhang et al. ${ }^{9}$ have proposed a class of PBG systems that are networks connected by segments of $1 \mathrm{D}$ waveguides. By introducing randomness into such systems, they have directly observed the Anderson localized wave functions inside the photonic band gap, despite the presence of dissipation. Excellent quantitative agreement between the theory and experiments has been obtained for both transmission coefficients and localized wave functions. There are two important advantages of the net- work system. First, strong scattering can be easily introduced in a unit cell to produce large full gaps in any dimension. Therefore unlike the usual PBG systems, these systems do not require a material with a large dielectric constant. Second, the wave function at each node is physically accessible so that $3 \mathrm{D}$ localized wave functions can be probed. On the same lines, Dobrzynski et al., ${ }^{10}$ Vasseur et al., ${ }^{11}$ and Pradhan and Watson ${ }^{12}$ have also reported interesting work.

Although photonic band gaps can be created in periodic networks, gaps may also exist in other network systems without periodicity, such as fractal networks. ${ }^{13-16}$ Due to their self-similar structures, fractals are much more complicated to study when compared with periodic systems. One of the main points of interest has been the fact that these selfsimilar objects are found to serve as a nontrivial model for the backbone of some transport problems. Fractals, in particular deterministic fractals, such as the Sierpinski gasket (SG) fractal, possess some special properties, one of which is scale invariance, and do not have any translational order. In fact they bridge the gap between periodic and disordered systems. In the past, the spectrum of eigenmodes as well as the wave functions for SGs have been extensively studied for both electronic systems and harmonic excitations. ${ }^{13-18}$ In such systems, there exist many structure-induced localized states, which are different in nature from the Anderson localized states that are caused by the multiple scattering of waves in a random environment. Besides localized states, there also exist an infinite number of extended states for an isolated SG. ${ }^{17,18}$ The effects of magnetic field on the electronic structure of SGs have also been studied. ${ }^{18}$ Liu and co-workers ${ }^{19,20}$ have developed a generalized eigenfunction method (GEM) to study the electronic transport properties of an open SG which is connected to electron reservoirs. The electronic localization induced by the fractal structure and by the quan- 
tum coherence effect has been systematically studied. The results of this work suggest that there could be photonic band gaps for the propagation of electromagnetic waves through Sierpinski networks (SNs). Motivated by this analogy, we choose to study the wave propagation and photonic band gap structure in SNs. Because the network equations of SNs can be mapped to the zero-energy single-electron tight-binding equation, we can use the GEM to numerically study the photonic band structure in the microwave range $0-500 \mathrm{MHz}$. We have calculated the transmission spectra for the first four generations of SNs. The fourth generation contains $N=123$ nodes. In each generation of SNs we consider both the cases of single and double exist. The effects due to dissipation are also studied. The numerical results display how the exit situation and dissipation effect can influence the magnitude of the transmission coefficient but not the photonic band structure. We have observed the interesting evolution of discrete eigenmodes as well as the photonic band gap structure following the enlargement of Sierpinski networks. We show explicitly that the resonant frequencies in a transmission spectrum coincide with the eigenfrequencies of the isolated $\mathrm{SN}$. We have also found an anomalous behavior of the transmission coefficient due to dissipation at the frequencies that correspond to $k=2 \pi n(n=0,1,2,3, \ldots)$, where $k$ is the wave vector in units of $\mathrm{m}^{-1}$. This anomaly can be explained by using a mathematical analysis. Since we have adopted two realistic parameters used in Ref. 9 for dissipation, all the results obtained here should also represent experimental measurements in a quantitative way.

This paper is organized as follows. In Sec. II, we derive the Sierpinski network equation and describe how to use the generalized eigenfunction method to calculate the transmission and reflection coefficients of Sierpinski networks. The numerical results and discussions of the photonic band structure as well as the transport properties of electromagnetic waves in SNs are presented in Sec. III. A brief summary is given in Sec. IV.

\section{NETWORK EQUATIONS AND GENERALIZED EIGENFUNCTION METHOD FOR SIERPINSKI WAVEGUIDE NETWORKS}

Following the work of Zhang et al., ${ }^{9}$ we use coaxial cables as 1D waveguides for the SNs. In Fig. 1, we show a fourth-generation SN that contains $N=123$ nodes. In general, we start with a network formed by waveguide segments of variable length. Each segment has a single channel for wave propagation. The electromagnetic wave function $\psi_{i j}$ within any segment between nodes $i$ and $j$ satisfies the following homogeneous wave equation: ${ }^{21}$

$$
\frac{\partial^{2} \psi_{i j}(s)}{\partial s^{2}}=\frac{\varepsilon \omega^{2}}{c_{0}^{2}} \psi_{i j}(s),
$$

where $\omega=2 \pi f$ is the frequency of the electromagnetic wave, $c_{0}$ is the electromagnetic wave speed in vacuum, and $s$ is the distance measured from node $i$. For a coaxial cable, $\psi$ denotes the voltage waves and $\varepsilon=\varepsilon_{0}+i \varepsilon^{\prime}$ is the relative permittivity of the dielectric medium. It is known that the solution of Eq. (1) has the form ${ }^{15,21}$

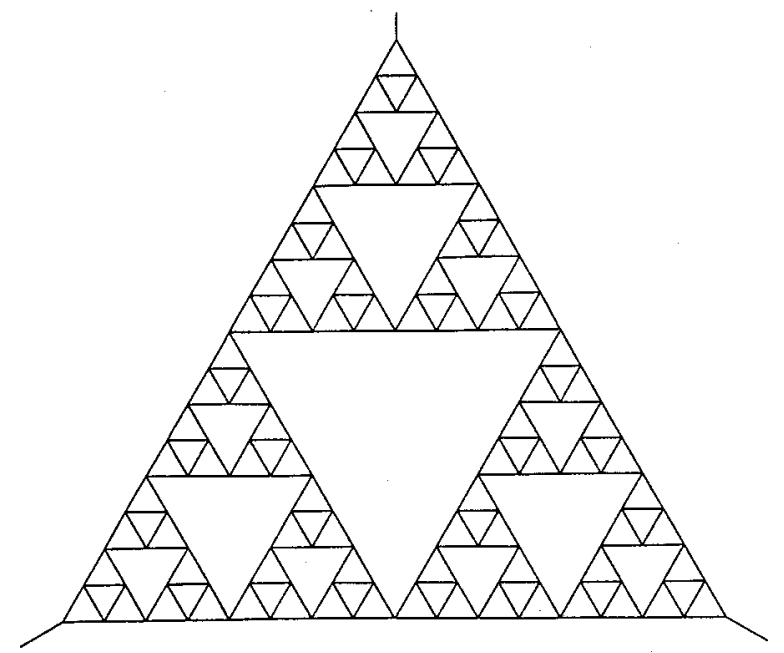

FIG. 1. The fourth-generation Sierpinski network, the electromagnetic wave transmission properties of which are studied in the text.

$$
\psi_{i j}(s)=\psi_{i} \frac{\sinh \left[\left(l_{i j}-s\right) z\right]}{\sinh \left(l_{i j} z\right)}+\psi_{j} \frac{\sinh (s z)}{\sinh \left(l_{i j} z\right)},
$$

where $l_{i j}$ is the length of the segment, $\psi_{i}$ and $\psi_{j}$ are, respectively, the values of the wave function at the nodes $i$ and $j$, and $z=\left(i \omega / c_{0}\right) \sqrt{\varepsilon}$. At each node $i$, the wave function is continuous and the derivative of the wave function at the node $i$ gives the following flux conservation condition:

$$
\sum_{j}\left[\frac{\partial}{\partial s} \psi_{i j}(s)\right]_{s=0}=0
$$

where the summation $j$ is over all the nodes linked directly to $i$. Substituting Eq. (2) into Eq. (3) we obtain the network equation

$$
-\psi_{i} \sum_{j} \operatorname{coth}\left(\theta_{i j}\right)+\sum_{j} \frac{1}{\sinh \left(\theta_{i j}\right)} \psi_{j}=0,
$$

where $\theta_{i j}=z l_{i j}$. If we compare Eq. (4) with the singleelectron tight-binding equation ${ }^{20,22}$

$$
\left(\varepsilon_{i}-E\right) \psi_{i}=\sum_{j} t_{i, j} \psi_{j}
$$

evidently Eq. (4) represents the zero-energy state with correlated site energy

$$
\varepsilon_{i}=\sum_{j} \operatorname{coth}\left(\theta_{i j}\right)
$$

and hopping matrix element

$$
t_{i j}=1 / \sinh \left(\theta_{i j}\right) .
$$

For this reason we can use the generalized eigenfunction method for the single-electron tight-binding equation to deal with the present electromagnetic wave transport problem. ${ }^{19}$ In the following we rewrite separately the network equation (4) in the absence and presence of dissipation. In the former case $\varepsilon^{\prime}=0, \varepsilon=\varepsilon_{0}$, so the wave vector $k=\omega \sqrt{\varepsilon_{0}} / c_{0}$. Equation (4) can be rewritten as 


$$
-\psi_{i} \sum_{j} \cot \left(\theta_{i j}\right)+\sum_{j} \frac{1}{\sin \left(\theta_{i j}\right)} \psi_{j}=0,
$$

where $\theta_{i j}=k l_{i j}$. Now we assume that all segments in the SN are of equal length, which is chosen as $l_{i j}=1(\mathrm{~m})$, for simplicity. In this case, the network equation (6) is reduced to

$$
-n a \psi_{i}+\sum_{j} \psi_{j}=0
$$

where $a=\cos k, k$ has units of $\mathrm{m}^{-1}$, and $n$ is the number of nearest-neighbor nodes.

In the presence of dissipation, we introduce a new parameter $z$,

$$
z=\frac{i \omega}{c_{0}} \sqrt{\varepsilon}=\frac{i \omega}{c_{0}} \sqrt{\varepsilon_{0}}\left(1+i \frac{\varepsilon^{\prime}}{\varepsilon_{0}}\right)^{1 / 2} .
$$

Because $\varepsilon^{\prime}<\varepsilon_{0}$, if only the first-order term of the Taylor expansion is kept, we have

$$
z=\frac{i \omega}{c_{0}} \sqrt{\varepsilon_{0}}\left(1+i \frac{\varepsilon^{\prime}}{2 \varepsilon_{0}}\right)=i k-\frac{k \varepsilon^{\prime}}{2 \varepsilon_{0}} .
$$

Further, we can introduce an absorption length $l=\varepsilon_{0} / k \varepsilon^{\prime}$ to simplify the above expression, i.e.,

$$
z=i k-\frac{1}{2 l}=i\left(k+\frac{i}{2 l}\right) .
$$

To simulate the experiment conditions ${ }^{9}$ we need to incorporate an additional dissipation due to connectors into the theoretical calculation. For this purpose we add a constant $\gamma$ to the terms $z l_{i j}$, i.e., we replace $z l_{i j}$ by $z l_{i j}+\gamma$. Thus, in the presence of dissipation, the network equation becomes

$$
-n(a+i b) \psi_{i}+\sum_{j} \psi_{j}=0
$$

where

$$
\begin{aligned}
& a=\frac{1}{2}\left(e^{\gamma-1 / 2 l}+e^{1 / 2 l-\gamma}\right) \cos k, \\
& b=\frac{1}{2}\left(e^{\gamma-1 / 2 l}-e^{1 / 2 l-\gamma}\right) \sin k .
\end{aligned}
$$

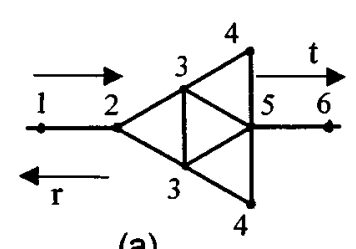

(a)

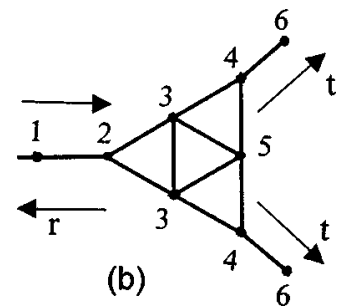

(b)

FIG. 2. First-generation Sierpinski network connected with a signal generator (entrance) and a spectrum analyzer (exit): (a) single-exit case; (b) double-exit case.

We start from the first-generation Sierpinski network with a single exit as shown in Fig. 2(a). In the absence of dissipation, Eq. (7) gives

$$
\begin{gathered}
3 a \psi_{2}-\psi_{1}-2 \psi_{3}=0, \\
4 a \psi_{3}-\psi_{2}-\psi_{3}-\psi_{4}-\psi_{5}=0 \\
2 a \psi_{4}-\psi_{3}-\psi_{5}=0 \\
5 a \psi_{5}-2 \psi_{4}-2 \psi_{3}-\psi_{6}=0 .
\end{gathered}
$$

On the other hand, for the special nodes at the entrance and the exit we can write down the wave functions as ${ }^{20}$

$$
\begin{gathered}
\psi_{1}-r=1, \\
\psi_{2}-r e^{-i k}=e^{i k}, \\
\psi_{5}-t=0, \\
\psi_{6}-t e^{i k}=0,
\end{gathered}
$$

where $r$ and $t$ are the reflection and transmission amplitudes of the outgoing waves, respectively. To numerically solve this problem we can use the generalized eigenfunction method which was developed to deal with the electronic transport problems of Sierpinski gasket lattices. ${ }^{19}$ The trick of the GEM lies in that we treat the amplitudes $r$ and $t$ like the wave functions $\psi_{i}$. In this way the coupled equations (9) and (10) can be rewritten as a matrix equation of order $N$ +2 , where $N$ is the number of nodes in the Sierpinski network:

$$
\left[\begin{array}{cccccccc}
-1 & 3 a & -2 & 0 & 0 & 0 & 0 & 0 \\
0 & -1 & (4 a-1) & -1 & -1 & 0 & 0 & 0 \\
0 & 0 & -1 & 2 a & -1 & 0 & 0 & 0 \\
0 & 0 & -2 & -2 & 5 a & -1 & 0 & 0 \\
1 & 0 & 0 & 0 & 0 & 0 & -1 & 0 \\
0 & 1 & 0 & 0 & 0 & 0 & -e^{-i k} & 0 \\
0 & 0 & 0 & 0 & 1 & 0 & 0 & -1 \\
0 & 0 & 0 & 0 & 0 & 1 & 0 & -e^{-i k}
\end{array}\right]\left[\begin{array}{c}
\psi_{1} \\
\psi_{2} \\
\psi_{3} \\
\psi_{4} \\
\psi_{5} \\
\psi_{6} \\
r \\
t
\end{array}\right]=\left[\begin{array}{c}
0 \\
0 \\
0 \\
0 \\
1 \\
e^{i k} \\
0 \\
0
\end{array}\right] .
$$


If we denote the above matrix equation as

$$
M \Psi=C,
$$

then the reflection and transmission amplitudes become

$$
\begin{aligned}
& r=\Psi_{7}=\left(M^{-1} C\right)_{7}, \\
& t=\Psi_{8}=\left(M^{-1} C\right)_{8} .
\end{aligned}
$$

The transmission and reflection coefficients are simply $T$ $=|t|^{2}$ and $R=|r|^{2}$, respectively. Evidently, the numerical solution of Eqs. (11)-(13) is easy to obtain even with a PC.

In the presence of dissipation, from Eq. (8), the network equation can be written as

$$
-\psi_{1}+3(a+i b) \psi_{2}-2 \psi_{3}=0,
$$

$\left[\begin{array}{cccc}-1 & 3(a+i b) & -2 & 0 \\ 0 & -1 & {[4(a+i b)-1]} & -1 \\ 0 & 0 & -1 & 2(a+i b) \\ 0 & 0 & -2 & -2 \\ 1 & 0 & 0 & 0 \\ 0 & 1 & 0 & 0 \\ 0 & 0 & 0 & 0 \\ 0 & 0 & 0 & 0\end{array}\right.$

The above matrix equation can again be denoted as $M \Psi$ $=C$, and the formal solutions for the reflection and transmission amplitudes remain the same as Eqs. (12) and (13). The generalized eigenfunction method is a very flexible approach to studying the transport property of a network system that contains large number of nodes. For example, in the absence of dissipation, the network equation and its corresponding matrix equation for the first generation of SN's with two exits, as is shown in Fig. 2(b), can be written as

$$
\begin{gathered}
-\psi_{1}+3 a \psi_{2}-2 \psi_{3}=0 \\
-\psi_{2}+(4 a-1) \psi_{3}-\psi_{4}-\psi_{5}=0
\end{gathered}
$$

$$
\begin{gathered}
-\psi_{2}+[4(a+i b)-1] \psi_{3}-\psi_{4}-\psi_{5}=0 \\
-\psi_{3}+2(a+i b) \psi_{4}-\psi_{5}=0 \\
-2 \psi_{3}-2 \psi_{4}+5(a+i b) \psi_{5}-\psi_{6}=0 \\
\psi_{1}-r=1 \\
\psi_{2}-r e^{-i k}=e^{i k} \\
\psi_{5}-t=0 \\
\psi_{6}-t e^{i k}=0
\end{gathered}
$$

The corresponding generalized eigenfunction matrix equation becomes

$$
\begin{gathered}
-\psi_{3}+3 a \psi_{4}-\psi_{5}-\psi_{6}=0, \\
-\psi_{3}-\psi_{4}+2 a \psi_{5}=0, \\
\psi_{1}-r=1, \\
\psi_{2}-r e^{-i k}=e^{i k}, \\
\psi_{5}-t=0 \\
\psi_{6}-t e^{i k}=0 .
\end{gathered}
$$

and

$$
\left[\begin{array}{cccccccc}
-1 & 3 a & -2 & 0 & 0 & 0 & 0 & 0 \\
0 & -1 & (4 a-1) & -1 & -1 & 0 & 0 & 0 \\
0 & 0 & -1 & 3 a & -1 & -1 & 0 & 0 \\
0 & 0 & -1 & -1 & 2 a & 0 & 0 & 0 \\
1 & 0 & 0 & 0 & 0 & 0 & -1 & 0 \\
0 & 1 & 0 & 0 & 0 & 0 & -e^{-i k} & 0 \\
0 & 0 & 0 & 1 & 0 & 0 & 0 & -1 \\
0 & 0 & 0 & 0 & 0 & 1 & 0 & -e^{-i k}
\end{array}\right]\left[\begin{array}{c}
\psi_{1} \\
\psi_{2} \\
\psi_{3} \\
\psi_{4} \\
\psi_{5} \\
\psi_{6} \\
r \\
t
\end{array}\right]=\left[\begin{array}{c}
0 \\
0 \\
0 \\
0 \\
1 \\
e^{i k} \\
0 \\
0
\end{array}\right] .
$$



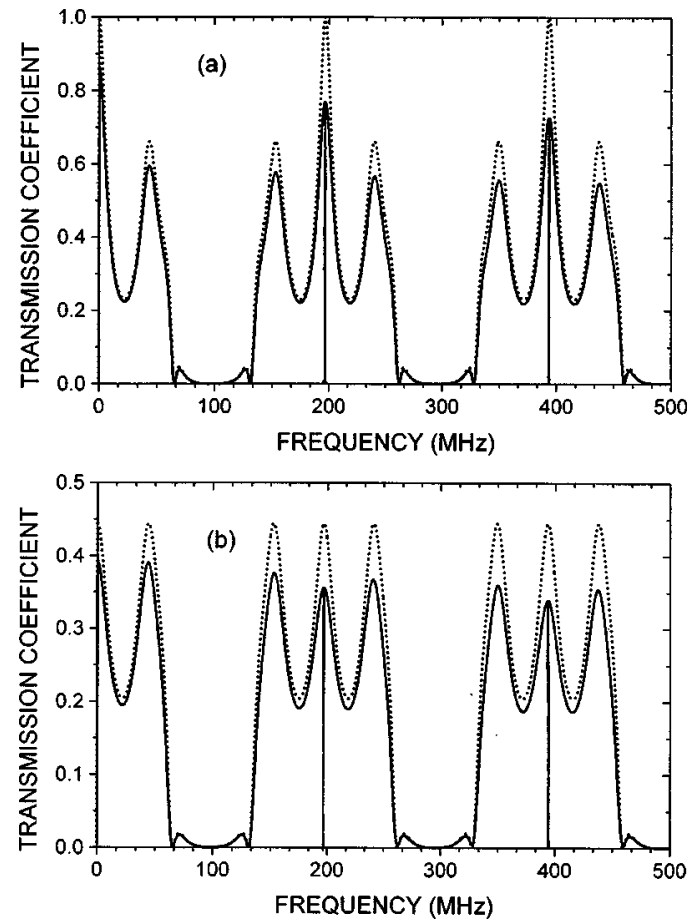

FIG. 3. Transmission coefficient $T$ as a function of frequency for the first-generation Sierpinski network. (a) For the case of a single exit, the solid and dotted curves are the results with and without dissipation, respectively. (b) The same as (a) for the case of double exits; here $T$ is the value in either one of the two exits.

respectively. For simplicity, in the above treatment we have used the symmetry between two exits. This treatment is rigorous, because there is no phase difference between two outgoing waves, which is different from the electronic transport properties in the presence of magnetic field. ${ }^{20,22}$ As in the single-exit case, if we denote the above matrix equation as $M \Psi=C$, we are able to obtain the reflection and transmission amplitudes from Eqs. (12) and (13). In this case the total transmission and reflection coefficients become $T=2|t|^{2}$ and $R=|r|^{2}$, respectively.

We would like to point out that it is easy to extend the above formalism to higher generations of SNs and other more complicated situations, such as with many entrances and exits or with disordered elements (segment length, structure). In the present article we use this GEM to calculate the transmission spectrum of SNs up to four generations for both single and double exits. To save space we will not write down the network equations and their corresponding matrix equation for the higher generations of SNs, but directly present the numerical results and discuss the electromagnetic wave transport properties in SNs.

\section{NUMERICAL RESULTS AND DISCUSSION}

The formalism presented in Sec. II can be easily implemented numerically and the results for both single and double exits have been obtained up to the fourth generation of SNs with $N=123$ nodes. With these four generations, we have already seen the main characteristics of wave transport in SNs. In order to make our results ready for experimental comparison $^{9}$ we have considered the dissipation effect by including the absorption length and the additional dissipation
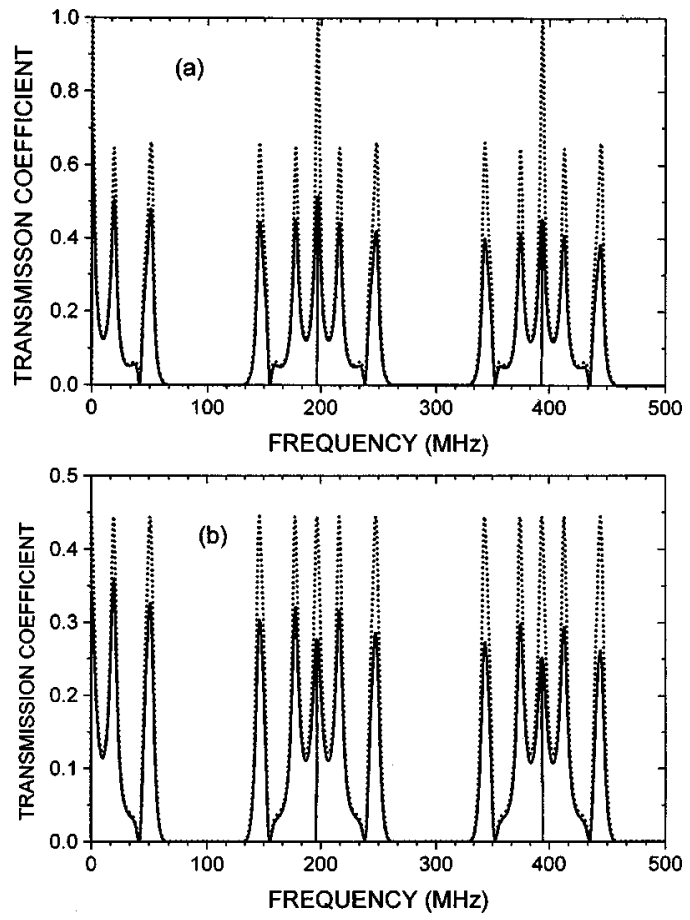

FIG. 4. The same as Fig. 3 for second-generation Sierpinski networks. Compared with Fig. 3, the antiresonant transmission dips (valleys) have now developed into photonic band gaps.

due to connectors. Following Ref. 9, we choose $l$ $\cong 1702 f^{-0.59}$, where $l$ is in units of $\mathrm{m}$ and $\gamma \cong-0.0075$. The value of $\varepsilon_{0}$ is taken as 2.32 . Thus, in the numerical calculation we can take

$$
k=\frac{2 \pi \sqrt{\varepsilon_{0}}}{2.997925} \frac{10^{6}}{10^{8}} f .
$$

The frequency range of interest here is $0-500 \mathrm{MHz}$. To examine the accuracy of our numerical calculations, we check at every intermediate stage of the calculation so that the criterion $T+R=1$ is satisfied to very high accuracy.

The main numerical results are shown in Figs. 3-8 below. The transmission spectra for the first generation are shown in Fig. 3. The transmission spectra for the second, third, and fourth generations are shown in Figs. 4, 5, and 6, respectively. In all of these figures the plots (a) are for the case of a single exit, and plots (b) are for the case of double exits. The solid and dotted curves represent the results with and without dissipation, respectively. From the numerical results obtained, we can observe interesting transmission properties as well as the photonic band gap structure, which are common for different generations of SNs. First, from Fig. 3 we observed that even in this simplest Sierpinski network with only six nodes photonic band gaps have appeared around 100 $\mathrm{MHz}$ in the first period. In the absence of dissipation, the transmission spectrum $T(f)$ (the dotted curve) is periodic with a period $196.8 \mathrm{MHz}$, which corresponds to $k$ $=2 \pi\left(\mathrm{m}^{-1}\right)$. The periodic structure in $T(f)$ is a result of a single length used for all waveguides in the entire network. This can also been seen from the network equation and transmission amplitude formulas (11) and (12), where $k$ appears in the form of $e^{i k}$. In the presence of dissipation (the solid curves), the reduction in the transmission coefficient in- 

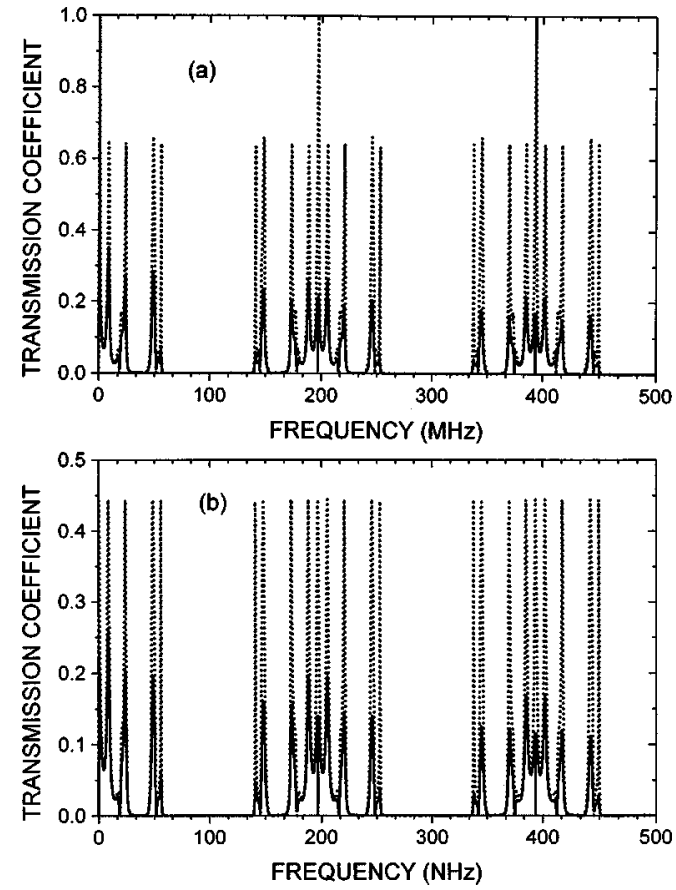

FIG. 5. Same as Fig. 3 for third-generation Sierpinski networks. Compared with Figs. 3 and 4, there are more photonic band gaps, and the dissipation effect has greatly depressed the transmission coefficient.

creases with the frequency. This is expected due to the frequency dependence of the absorption length, i.e., $l \sim k^{-0.59}$. By comparing Figs. 3(a) and 3(b), it is easy to see that the position of the resonant peak does not depend on the exit situation. This is expected as these peak positions represent the eigenfrequencies of the system and should not be sensitive to the exit situation. They should also not be much altered by the presence of modest dissipation. A direct verification of this point will be made later.

In the absence of dissipation, the difference caused by the exit situation lies in the magnitude of $T$. In the case of a single exit [Fig. 3(a)], the largest resonant peaks are located at $f=0$ as well as other periodic positions with $T=1$. This is also expected if we consider the entire $\mathrm{SN}$ as a single scatterer in a one-dimensional system. In the long-wavelength limit, the scattering becomes weak and $T$ approaches unity. However, this is no longer true for the case of double exits [Fig. 3(b)], where all the resonant peaks have the same magnitude $|t|_{\max }^{2}=0.45$ for either one of the two exits. The total transmission has a maximum value $T_{\max }=0.9$ even in the zero-frequency limit. As for the transmission at other resonant frequencies, the total transmission of double exits is larger than that of a single exit. This point can also be observed in the higher-generation SNs. An intuitive explanation for this phenomenon could be that for the case of double exits, waves can propagate directly from the entrance to the two exits through straight and direct channels, whereas, in the case of a single exit, more waves will be reflected at the two corners on the output side.

Following the enlargement of the Sierpinski network, i.e., for the higher generations of SNs, the transmission spectra become more complicated. Comparing the results of the first four generations of SNs shown in Figs. 3-6, we can observe the following phenomena.
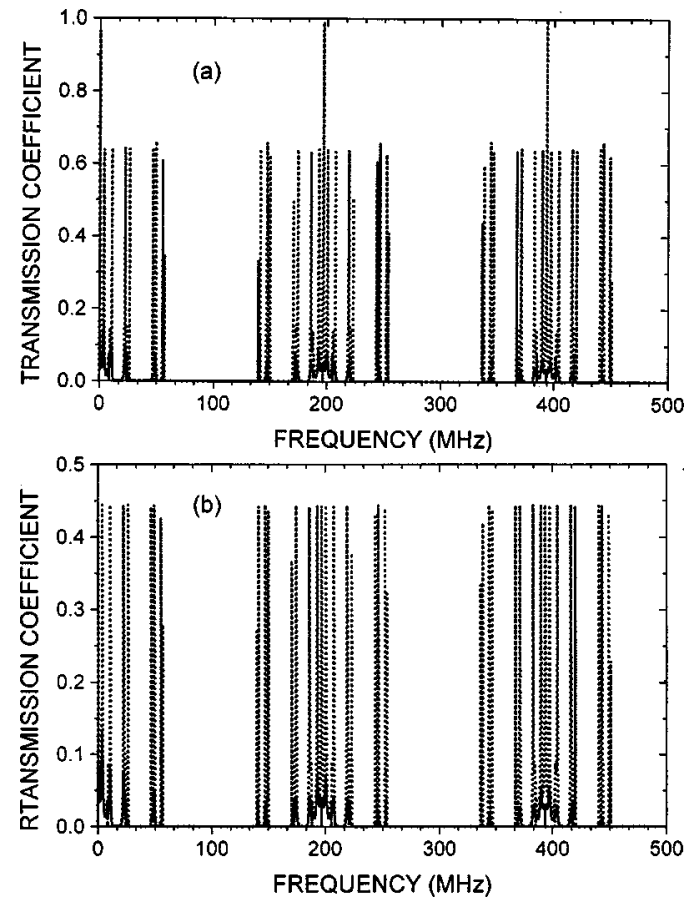

FIG. 6. Same as Fig. 3 for fourth-generation Sierpinski networks. More $\delta$-like resonant transmission peaks and photonic band gaps have developed. The dissipation effect has drastically depressed the transmission coefficient.

(1) The number of resonant transmission peaks progressively increases, from three peaks in a period for the first generation to fifteen peaks for the fourth generation, the peaks also becoming sharper and sharper. It is easy to understand these results. As the size of the $\mathrm{SN}$ is increased, there are more nodes in the system. Stronger multiple scattering allows more states with wavelengths smaller than the sample size to appear. Also, the stronger multiple scattering increases the dwell time of a resonant state and, therefore, makes the peak sharper. As a consequence, sharper resonant peaks as well as antiresonant dips (valley shape) will develop into photonic band gaps in the higher generation of SNs. This phenomenon can be clearly seen if we compare Fig. 4 with Fig. 3. Also, since the number of resonant states increases with system size, more photonic band gaps appear in the higher generations of SNs. These resonant peaks become $\delta$-like functions in the higher generations as can be seen in Fig. 6. Thus, we have observed the evolution and the appearance of more discrete eigenmodes as the size of the SN is increased. However, the main gap centered around $100 \mathrm{MHz}$ remains unchanged.

(2) Following the enlargement of the SNs, the influence of dissipation effects on the transmission is also increased. The transmission coefficient $T$ decreases more drastically, i.e., from $T_{\max }$ around 0.6 at the first generation to $T_{\max }<0.1$ at the fourth generation. This is reasonable because, in a larger $\mathrm{SN}$, the electromagnetic wave undergoes longer path lengths and is scattered by more nodes, both of which enhance the dissipation effect.

The above discussion describes the main properties of electromagnetic wave propagation in Sierpinski waveguide networks. Here we would like to ask two more questions. First, would the eigenfrequencies of an isolated SN give the 


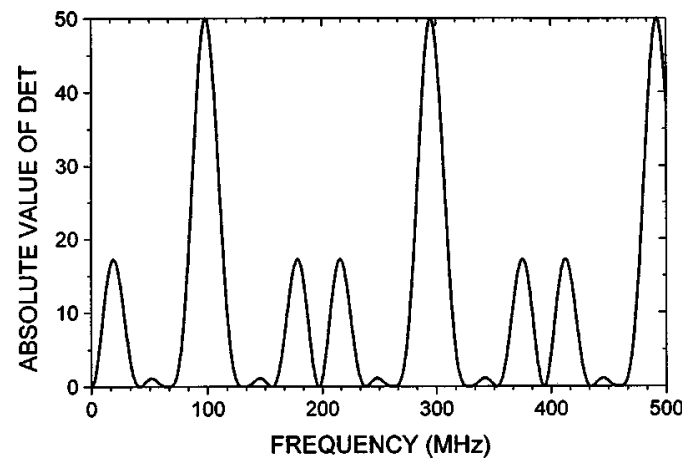

FIG. 7. Determinant $\mathbf{M}$ as a function of frequency for the firstgeneration Sierpinski network. Comparing this figure with Fig. 3, it is clear that the eigenfrequencies of isolated Sierpinski networks coincide with the frequencies of resonant transmission peaks.

same photonic band structures as that obtained from the transmission study? We have noticed that the photonic band structure is independent of the exit number and dissipation effects, which lets us guess that the photonic band structure should be related to an intrinsic property of the Sierpinski networks. To check this, as an example, we investigate the eigenmode spectrum of an isolated first-generation $\mathrm{SN}$, which is shown in Fig. 2 but without connecting waveguides at entrance and exits. For this isolated SN network, the matrix equation can be written as

$$
\left[\begin{array}{cccccc}
2 a & -1 & -1 & 0 & 0 & 0 \\
-1 & 4 a & -1 & -1 & -1 & 0 \\
-1 & -1 & 4 a & 0 & -1 & -1 \\
0 & -1 & 0 & 2 a & -1 & 0 \\
0 & -1 & -1 & -1 & 4 a & -1 \\
0 & 0 & -1 & 0 & -1 & 2 a
\end{array}\right]\left[\begin{array}{l}
\psi_{1} \\
\psi_{2} \\
\psi_{3} \\
\psi_{4} \\
\psi_{5} \\
\psi_{6}
\end{array}\right]=\left[\begin{array}{l}
0 \\
0 \\
0 \\
0 \\
0 \\
0
\end{array}\right] .
$$

The above matrix equation can be written as $\mathbf{M} \psi=\mathbf{0}$. We numerically evaluated the determinant $|\mathbf{M}|$ as a function of frequency $f$ and show the result in Fig. 7. The frequency where the determinant vanishes corresponds to an eigenfrequency $f_{\text {eig }}$ of the SN. By comparing Fig. 3 with Fig. 7, we do find that the resonant frequencies coincide with the eigenfrequencies $f_{\text {eig }}$. This confirms that the photonic band struc-
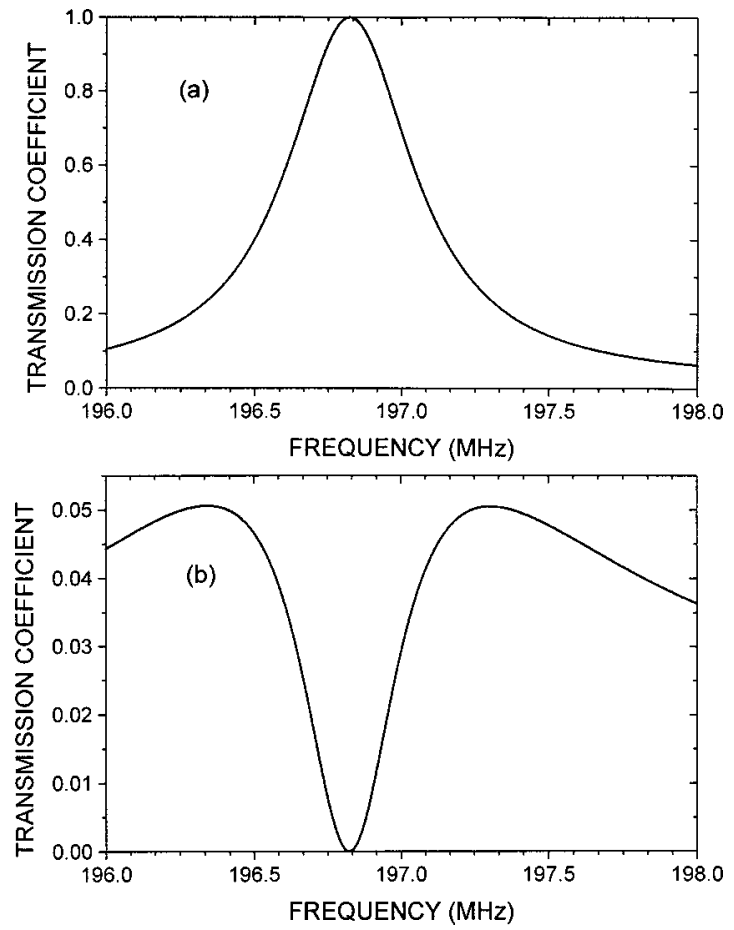

FIG. 8. An enlarged picture of Fig. 6 around frequency 196 $\mathrm{MHz}$ for the fourth-generation Sierpinski network with a single exit: (a) for the case without dissipation, and (b) for the case with dissipation.

tures obtained from transmission spectra are dictated by the eigenmodes of the system and are independent of the exit situation and dissipation effects. The second question is that in Figs. 3-6 we have noticed a phenomenon caused by dissipation, i.e., at frequencies $f \cong n \times 196 \mathrm{MHz}$, which correspond to $k=2 n \pi(n=0,1,2, \ldots)\left(\mathrm{m}^{-1}\right)$. The transmission coefficient has a double-peak structure with a $T=0$ valley, when dissipation is included. In the absence of dissipation there is only a single peak with $T=1$. We enlarge this portion of $T(f)$ for the fourth generation of SNs and plot it in Fig. 8. To understand the cause of this splitting, we use the following mathematical analysis. When $k=2 n \pi \quad(n$ $=0,1,2, \ldots)\left(\mathrm{m}^{-1}\right)$, we have $b=0, e^{i k}=1$, and the matrix equation (14) reduces to the following homogeneous matrix equation:

$$
\left[\begin{array}{cccccccc}
-1 & 3 a & -2 & 0 & 0 & 0 & 0 & 0 \\
0 & -1 & (4 a-1) & -1 & -1 & 0 & 0 & 0 \\
0 & 0 & -1 & 2 a & -1 & 0 & 0 & 0 \\
0 & 0 & -2 & -2 & 5 a & -1 & 0 & 0 \\
1 & 0 & 0 & 0 & 0 & 0 & -1 & 0 \\
0 & 1 & 0 & 0 & 0 & 0 & -1 & 0 \\
0 & 0 & 0 & 0 & 1 & 0 & 0 & -1 \\
0 & 0 & 0 & 0 & 0 & 1 & 0 & -1
\end{array}\right]\left[\begin{array}{c}
\psi_{1} \\
\psi_{2} \\
\psi_{3} \\
\psi_{4} \\
\psi_{5} \\
\psi_{6} \\
r+1 \\
t
\end{array}\right]=\left[\begin{array}{c}
0 \\
0 \\
0 \\
0 \\
0 \\
0 \\
0 \\
0
\end{array}\right] .
$$


It is easy to show that the determinant of $\mathbf{M}$ has the form $|\mathbf{M}|=(a-1)\left(120 a^{3}+26 a^{2}-21 a-5\right)$. In the case of no dissipation, since $a=\cos k=1,|\mathbf{M}|$ equals zero and there exists a nontrivial solution. However, in the presence of dissipation, $a \neq 1$, as can be seen from Eq. (8), and $|\mathbf{M}| \neq 0$. In this case, there exists no nontrivial solution. This implies $r=$ -1 and $t=0$, or $T=0$. This explains the splitting of a peak into two with a $T=0$ valley in the middle as is shown in Fig. 8. This splitting is due to an intrinsic property of SNs and is not related to the exit situation. Therefore, similar splitting appears in the case of double exits as is shown in Figs. 3(b)6(b).

\section{BRIEF SUMMARY}

The main purpose of the present article is to study the photonic band structure of Sierpinski networks consisting of $1 \mathrm{D}$ waveguides. The existence of photonic band gaps is a result of the self-similar structure, not due to periodicity. Because the present network equation can be mapped to the zero-energy single-electron tight-binding equation, it can be numerically solved by using the generalized eigenfunction method. We have investigated the wave transport properties of the SN for the first four generations. Both cases of single and double exits have been considered. To simulate the experimental conditions we have also investigated the dissipation effect by introducing a realistic absorption length and the additional dissipation due to connectors. Since all the segments in the network are of the same length $(1 \mathrm{~m})$, the transmission spectrum is periodic with a period $196.8 \mathrm{MHz}$. The effect of dissipation is to decrease the transmission spectra in a systematic way. The structures in the transmission spectra are found to be insensitive to the exit situation as well as to the presence of dissipation. They are closely related to the eigenfrequencies of isolated SNs.

Following the enlargement of the Sierpinski network, the number of resonant peaks progressively increases and the photonic band structures become complicated. Both the number and depth of the antiresonant transmission dips (valleys) are increased; these valleys will develop into photonic band gaps in the higher generations of SNs. On the other hand, following the enlargement of the SN system the influence of dissipation effects on the transmission coefficient is enhanced. One interesting effect of dissipation is to split the resonant peaks that correspond to $k=2 \pi n\left(\mathrm{~m}^{-1}\right)$ into two peaks with zero transmission in the valley. An analytic expression is used to explain this anomaly.

\section{ACKNOWLEDGMENTS}

This work was supported by the National Natural Science Foundation of China, Project No. 19874021. Z. Q. Zhang acknowledges support from Research Grant No. HKUST 6112/98p.
*Mailing address.

${ }^{1}$ See, for example, Scattering and Localization of Classical Waves in Random Media, edited by P. Sheng (World Scientific, Singapore, 1990).

${ }^{2}$ For example, Photonic Band Gap Materials, edited by C. M. Soukoulis (Kluwer, Dordrecht, 1995).

${ }^{3}$ S. John, Phys. Rev. Lett. 58, 2486 (1987).

${ }^{4}$ E. Yablonovitch, Phys. Rev. Lett. 58, 2059 (1987).

${ }^{5}$ D. Z. Zhang, W. Wu, Y. L. Zhang, L. Li, B. Y. Cheng, and G. Z. Yang, Phys. Rev. B 50, 9810 (1994).

${ }^{6}$ S. L. McCall, P. M. Platzman, R. Dalichaouch, D. Smith, and S. Schultz, Phys. Rev. Lett. 67, 2017 (1991); Nature (London) 354, 53 (1991)

${ }^{7}$ A. Z. Genack and N. Garcia, Phys. Rev. Lett. 66, 2064 (1991); 66, 1850 (1991).

${ }^{8}$ D. S. Wiersma, P. Bartolini, Ad. Lagendijk, and R. Righini, Nature (London) 390, 671 (1997).

${ }^{9}$ Z. Q. Zhang, C. C. Wong, K. K. Fung, Y. L. Ho, W. L. Chan, S. C. Kan, T. L. Chan, and N. Cheung, Phys. Rev. Lett. 81, 5540 (1998).
${ }^{10}$ L. Dobrzynski, A. Akjouj, B. Djafari-Rouhani, and J. O. Vasseur, Phys. Rev. B 57, R9388 (1998).

${ }^{11}$ J. O. Vasseur, B. Djafari-Rouhani, L. Dobrzynski, and A. Akjouj, Phys. Rev. B 59, 13446 (1999).

${ }^{12}$ R. D. Pradhan and G. H. Watson, Phys. Rev. B 60, 2410 (1999).

${ }^{13}$ E. Domany, S. Alexander, D. Bensimon, and L. P. Kadanoff, Phys. Rev. B 28, 3110 (1983).

${ }^{14}$ R. Rammal and G. Thoulous, Phys. Rev. Lett. 49, 1194 (1982).

${ }^{15}$ M. Alexander, Phys. Rev. B 27, 1591 (1983).

${ }^{16}$ A. Lakhtakia, R. Messier, V. K. Varadan, and V. V. Varadan, Phys. Rev. A 34, 2501 (1986).

${ }^{17}$ A. Chakrabarti, J. Phys.: Condens. Matter 8, 10951 (1996).

${ }^{18}$ X. R. Wang, Phys. Rev. B 53, 12035 (1998); 51, 9310 (1995).

${ }^{19}$ Youyan Liu, Zhilin Hou, P. M. Hui, and W. Sritrakool, Phys. Rev. B 60, 13444 (1999).

${ }^{20}$ Jinbo Li, Z. Q. Zhang, and Youyan Liu, Phys. Rev. B 55, 5337 (1997).

${ }^{21}$ Z. Q. Zhang and P. Sheng, Phys. Rev. B 49, 83 (1994).

${ }^{22}$ Youyan Liu, Honglin Wang, Z. Q. Zhang, and Xiujun Fu, Phys. Rev. B 53, 6943 (1996). 